\title{
MEMS-based Heating Element for in-situ Dynamical Experiments on FIB/SEM Systems
}

\author{
Libor Novák $^{1}$, Jaroslav Stárek ${ }^{1}$, Tomáš Vystavěl ${ }^{1}$ and Luigi Mele ${ }^{2}$ \\ ${ }^{1}$ FEI Company, Vlastimila Pecha 12, 62700 Brno, Czech Republic \\ ${ }^{2}$ FEI Company, Achtseweg Noord 5, 5600 KA Eindhoven, The Netherlands
}

In-situ observation of microstructural evolution of solids such as recrystallization, grain growth and phase changes in SEM is important for various fields of material science and industry research. This technique requires reliable discrimination of differently oriented crystal phases combined with useful spatial and temporal resolution and with fast and precise control of specimen temperature. While the requirements on spatial and temporal resolution are satisfied by current SEMs with resolution below $1 \mathrm{~nm}$ and $100 \mathrm{~Hz}$ frame rate, existing heating holders for bulk samples only allow for heating rates up to $300^{\circ} \mathrm{C}$ per minute $\left(5^{\circ} \mathrm{C} / \mathrm{s}\right)$. Long ramping time, which is required during heating experiments done using these devices, may cause unwanted sample changes (e.g. oxidation or recrystallization) before the temperature range of interest is reached. Thermal radiation of massive heating holders decreases quality of material contrast imaging as the commonly used detectors of backscattered electrons become saturated by thermally emitted photons. MEMS-based heating holder [1], [2] in combination with in-situ site specific sample preparation using a FIB/SEM system brings significant improvement in instrumentation for in-situ heating experiments inside the SEM chamber.

Low heated mass achieved by MEMS technology and heating control integrated into FIB/SEM system enable the user to perform dynamical experiments with heating rate in the order of $10^{5}{ }^{\circ} \mathrm{C} / \mathrm{s}$. The design of the MEMS heating chip and its controller assure temperature stability within $1^{\circ} \mathrm{C}$ after only few tens of miliseconds from heating start. At $1000^{\circ} \mathrm{C}$, the central heating plate shows good temperature uniformity with less than $1 \%$ deviation from the target temperature [1]. The MEMS chip can be repeatedly heated up to $1300^{\circ} \mathrm{C}$ in vacuum conditions without performance loss [1]. Very low heating power of several $\mathrm{mW}$ does not bring limitation to the detection of any FIB/SEM signal (SE, BSE, secondary ions) including characteristic X-rays and EBSD [4] (Figure 1). STEM imaging is possible trough dedicated perforation in homogeneously heated area of the microheater. Besides rapid heating and cooling the chip enables sample biasing and resistance measurement using 4-point probe method. Uniformly heated area with diameter of $100 \mu \mathrm{m}$ is covered by thin silicon nitride shell protecting metal parts of the chip during long-term high temperature experiments in presence of oxidizing environment.

Workflow for site-specific bulk sample preparation in a SEM/FIB system was developed [3]. A chunk of material is first cut with the FIB and attached to the micromanipulator needle using beam-induced deposition (Figure 2a). After lift-out the sample can be shaped on the needle using the FIB (2b). Then it is placed on the MEMS heating holder (2c), fixed with beam-induced deposition and cut loose from the needle. The sample surface can be then optionally cleaned by FIB in order to reveal sub-surface features. Standard SEM holder with bulk sample, from which the selected chunk is taken, can be placed on dedicated SEM stage together with MEMS heater unit. This configuration enables sample mounting to MEMS without chamber venting and minimizes risk of irrecoverable surface oxidation or contamination before the heating experiment. MEMS chip is connected to the controller during these steps and can be kept at elevated temperature already during sample mounting. 


\section{References:}

[1] L. Mele et al. A MEMS-based heating holder for the direct imaging of simultaneous in-situ heating and biasing experiments in scanning/transmission electron microscopes, Microscopy Research and Technique, Published online 28-Jan 2016, DOI: 10.1002/jemt.22623

[2] L. Mele et al. A molybdenum MEMS microhotplate for high-temperature operation, Sensors \& Actuators: A. Physical, 2012 | 188 | 173-180

[3] L. Novak et al. MEMS element for in-situ heating experiments on FIB/SEM systems, FEBIP 2014 Abstract Book, p. 199-200

[4] The authors acknowledge funding from the Technology Agency of the Czech Republic: project TE01020118 and A. Patak and F. Mika from Institute of Scientific Instruments of the CAS for assistance with part of experiments.
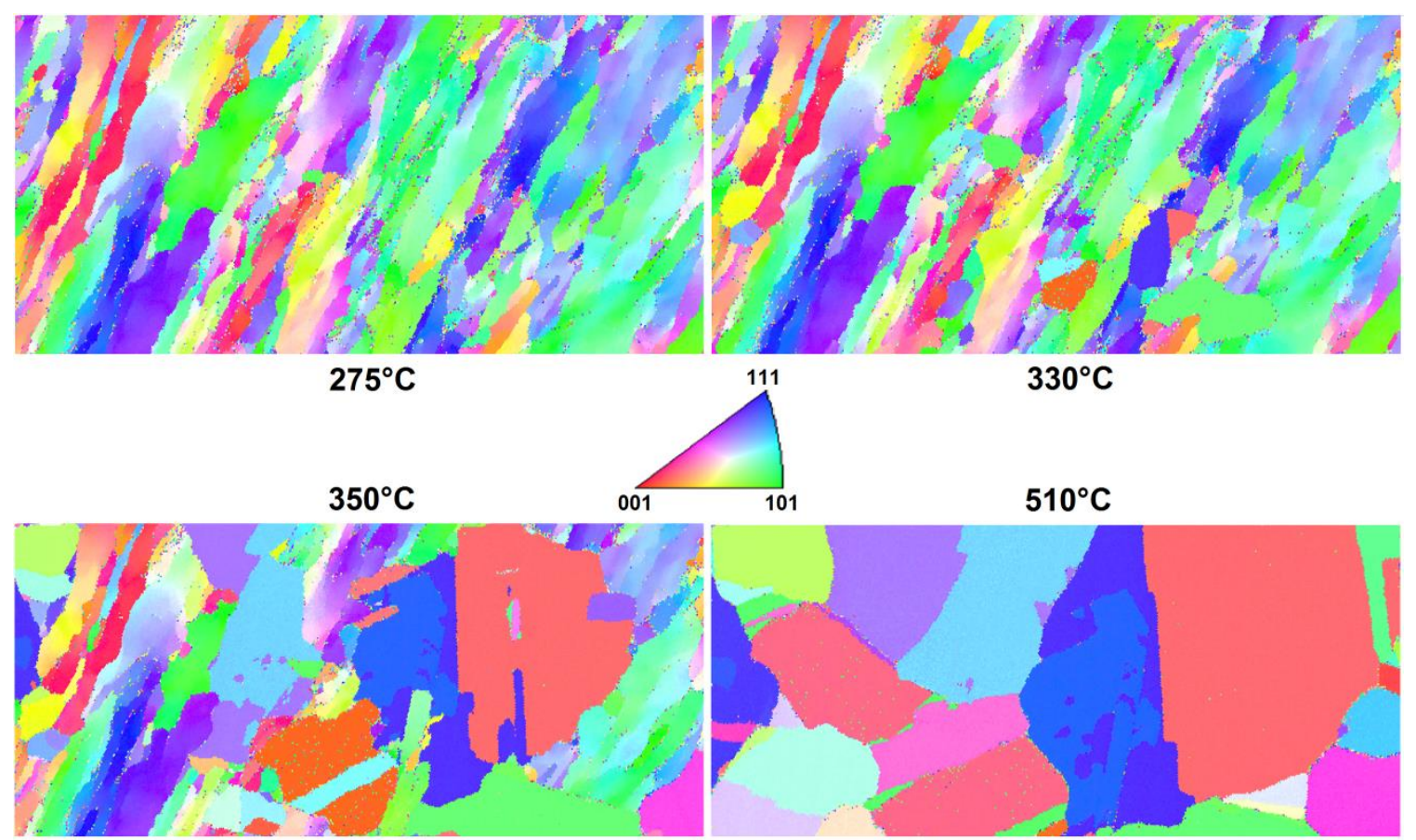

Figure 1. Recrystallization of ECAP copper placed on MEMS heater (EBSD imaging). Horizontal field width is $16 \mu \mathrm{m}$.

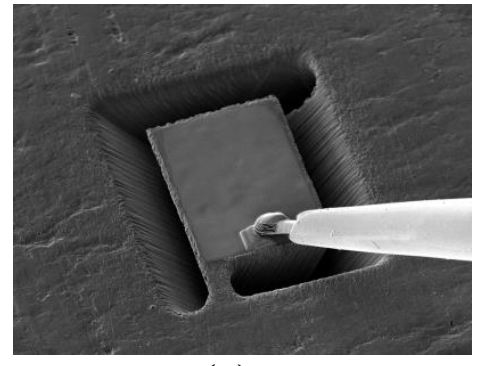

(a)

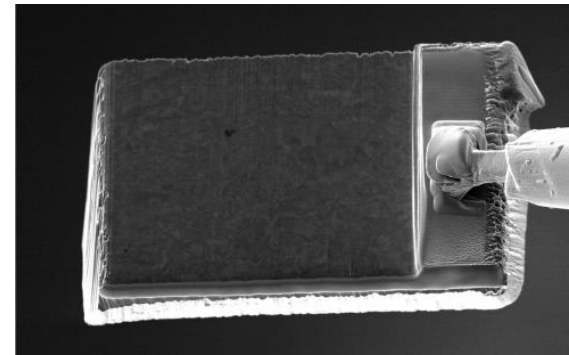

(b)

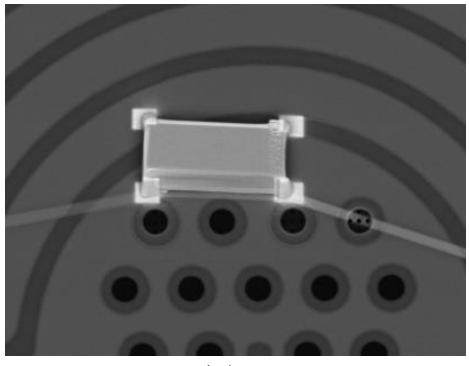

(c)

Figure 2. Sample preparation from bulk sample: (a) extraction using ion beam and manipulator; (b) shaping of sample on manipulator needle; (c) Pt deposition used for sample fixation to MEMS and for sample biasing (Pt wires leading to opposite biasing pads located outside heated area). Horizontal field widths are $90 \mu \mathrm{m}(\mathrm{a}), 50 \mu \mathrm{m}$ (b), and $100 \mu \mathrm{m}$ (c). 